\title{
氭化叶绿素类二氢卟吩衍生物的合成及其光动力活性的研究
}

\author{
高 娜 ${ }^{a}$ 王 振 ${ }^{b}$ 武 进 ${ }^{b}$ 刘 超 ${ }^{b}$ 王进军 $*, a, b$ \\ ( ${ }^{a}$ 烟台大学文经学院食品与生物工程系 烟台 264005) \\ ( ${ }^{b}$ 烟台大学化学化工学院 烟台 264005)
}

\begin{abstract}
摘要 以焦脱镁叶绿酸-a 甲酯为起始原料, 选择硝酸铊和空气为氧化剂对其进行氧化, 分别在二氢卟吩周环上的 3,12 和 15 位上引进了甲酰基和甲酰甲基，与盐酸羟胺肜化后，在 $N, N$-二甲基甲酰胺和三氯三嗪的促进下脱水并顺利地转化 为氰基; 利用丙二腈与二氢卟吩环上羰基的瑙文格尔反应建立了二氰亚甲基结构, 进一步的结构修饰则在色基上完成 了多氰基取代. 通过叶绿素降解产物周环上的不同位置实施氰基化，合成了一系列未见报道的氧基取代的叶绿素类二 氢卟吩衍生物, 其化学结构均经 UV、IR、 ${ }^{1} \mathrm{H}$ NMR 及元素分析予以证实; 同时也讨论了叶绿素类二氢卟吩氰基化的反 应机理, 并对部分新化合物的体外光动力活性进行了测试.
\end{abstract}

关键词 叶绿素-a; 二氢卟吩; 化学修饰; 氰基化反应; 光动力活性

\section{Synthesis of Cyanided Chlorophyllous Chlorin Derivatives and Study on Their Photodynamic Activities}

\author{
Gao, $\mathrm{Na}^{a} \quad$ Wang, Zhen ${ }^{b} \quad \mathrm{Wu}$, Jin $^{b} \quad$ Liu, Chao $^{b} \quad$ Wang, Jinjun ${ }^{*, a, b}$ \\ ( ${ }^{a}$ Department of Food \& Biological Engineering, Wenjing College, Yantai University, Yantai 264005) \\ ( ${ }^{b}$ College of Chemistry and Chemical Engineering, Yantai University, Yantai 264005)
}

\begin{abstract}
Pyropheophorbide-a methyl ester was used as a starting material, and its oxygenations were carried out by thaillum nitrate and air as oxidizing agent to introduce the formyl group and the formylmethyl group at 3-, 12- or 15-postion of chlorin periphery, respectively. After oximation these aldehyde groups were converted into cyano group under the reaction mediated from $N, N$-dimethylformamide and 2,4,6-trichloro-1,3,5-triazine. Dicyanomethylene moiety was established by Knoevenagel reaction of malononitrile with formyl group and $13^{1}$-carbounyl group on the exocyclic ring. Mulcyano-substitutions on the chromophore were accomplished by further structure modifications. A series of unreported chlorins related to chlorophyll substituted with cyano-group were synthesized making use of cyanation at different positions on the periphery of chlorophyll degradation products. Their chemical structures were characterized by elemental analysis, UV, IR and ${ }^{1} \mathrm{H}$ NMR spectra. The reaction mechanisms on the cyanation for the chlorophyllous chlorins were discussed and in vitro photodynamic activities of some new compounds were determined.
\end{abstract}

Keywords chlorophyll-a; chlorin; chemical modification; cyanation reaction; photodynamic activity

在叶绿素类光动力治疗 (photodynamic therapy, 简 称 PDT)药物的合成研究中, 许多定量构效关系(QSAR) 的研究表明, 叶绿素类二氢卟吩环上取代基的几何形 状、电荷密度以及连接的位置对 PDT 活性都将产生深 刻的影响, 特别是特定的官能结构往往对四吡咯大环分 子的理化、光物理和光生物性质起到至关重要的作用, 而这些数据正是表征光动力抗癌活性的重要参数 ${ }^{[1,2]}$.
因此, 在叶绿素周环上引进和建立活性反应区域是设计 理想 PDT 光敏剂的基本合成策略和重要的先期工作 ${ }^{[3,4]}$. 根据碳氮参键的直线结构特征、非对称的电荷密度以及 活泼的化学反应性质, 无论从空间结构、电子效应还是 结构转换上考虑, 氧基单元的存在都有可能使得四吡咯 大环发生不同程度的变化. 本文基于课题组和他人的前 期研究基础 ${ }^{[5 \sim 7]}$, 选择焦脱镁叶绿酸-a 甲酯(MPPa, 1)为

*E-mail: wjj1955@163.com

Received June 30, 2015; revised June 1, 2015; published online November 16, 2015.

Project supported by the National Natural Science Foundations of China (No. 21272048) and the Project of Shandong Applied Reasearch Centre of Gold Nanotechnology (2011).

国家自然科学基金(No. 21272048)和山东省黄金工程技术研究中心(2011 年度)资助项目. 
起始原料, 通过化学转换, 在四吡咯周环上的不同位置 上构建了碳氮参键，合成出一系列未见报道的氰基取代 的焦脱镁叶绿酸-a 甲酯衍生物, 为寻找新型 PDT 光敏剂 提供了具有更多反应位点的合成前体.

选择硝酸铊和空气对焦脱镁叶绿酸-a 甲酯(1)实施 氧化, 分别得到 3-甲酰甲基焦脱镁叶绿酸-a 甲酯(2)、15甲酰基二氢卟吩- $f$ 二酯(3)和 12-甲酰基焦脱镁叶绿酸-a 甲酯(4). 在室温条件下, 二氢卟吩醛 2 在甲醇溶液中与 盐酸着胺摚拌反应 $1 \mathrm{~h}$, 以 $72 \%$ 的产率得到二氢卟吩醛 肜(5); 选择 $N, N$-二甲基甲酰胺作为反应溶剂, 并与三氯 三嗪(TCT)协同促成脱水反应, 将 3-位肜基转变为氰基, 分离出 48\%的 3-氰甲基焦脱镁叶绿酸-a 甲酯(6). 3 成肜 后不经分离, 直接进行相应的脱水反应, 以 $26 \%$ 的总收 率得到 15-氰基二氢卟吩- $f$ 二酯(7); 二氢卟吩醛 4 与盐 酸着胺在 $N$-甲基吡咯烷酮中相互作用，一步完成肟氰 转换, 并收集到 29\%的 12-氰基焦脱镁叶绿酸-a 甲酯 $(8)$ (Scheme 1).

在含有三乙胺的四氢呋喃溶液中, 二氢卟吩醛 4 与 丙二腈顺利地发生瑙文格尔反应(Knoevenagel reaction), 以理想的产率生成二氰基取代的叶绿素衍生物 9 (75\%); 尝试在含有甲醇钠的甲醇溶液中对外接环羰基继续实
施二氰亚甲基化，结果分离出四氰基取代的二氢卟吩 $10(28 \%)$ 和二氰基取代的二氢卟吩 $11(8 \%)$; 如果先行 将外接环羰基与丙二腈进行缩合，很容易得到 $13^{1}$-二氰 亚甲基焦脱镁叶绿酸-a 甲酯(12), 直接在碱性条件下对 其进行空气氧化, 期望在周环上建立新的甲酰基结构, 但只从复杂的混合物中分离出少量的二氧基取代的叶 绿素衍生物 13 (18\%)和 14 (4\%) (Scheme 2).

\section{1 结果与讨论}

\section{1 叶绿素降解产物周环上的氰基形成过程}

肜醛基的贝克曼重排经常作为合成腈类化合物的 经典方法, 其结构转换需要脱去一分子水, 因此, 如何 促进羟基的离去则是完成碳氮参键形成的关键. 霍夫曼 重排一般需要较高的反应温度、较强的酸性和无水的反 应介质，因而该反应往往导致大量的副产物生成，极大 地受限于在敏感性反应底物中的应用. 所以，寻找温和 的反应条件完成贝克曼重排，一直是有机合成领域中的 热点研究内容. 考虑到叶绿素衍生物对酸、热和光等环 境的敏感性, 基于本室与他人有关贝克曼重排的前期工 作 ${ }^{[8 \sim 10]}$, 选择三氯三嗪和 $N, N$-二甲基甲酰胺(DMF)共同

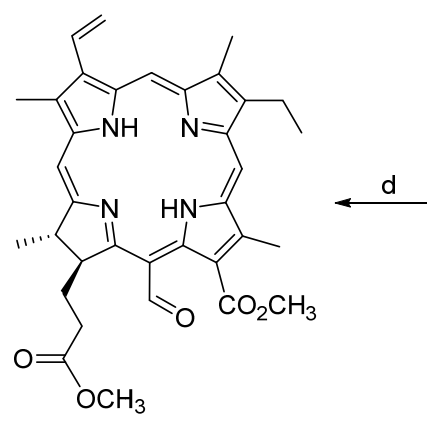

3

$\downarrow b+c$

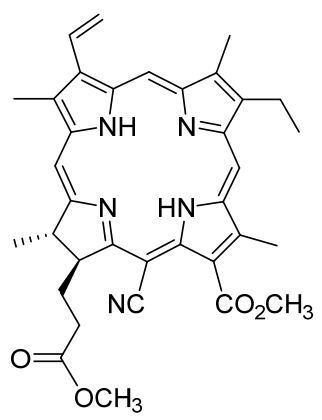

7

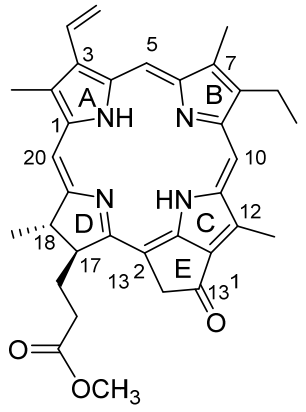

1

$\downarrow d$

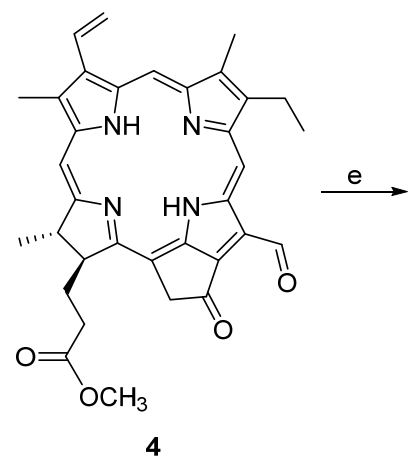

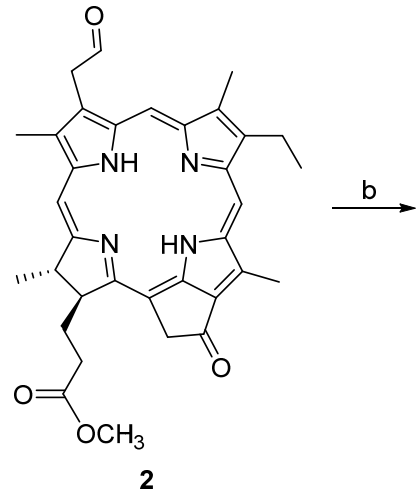

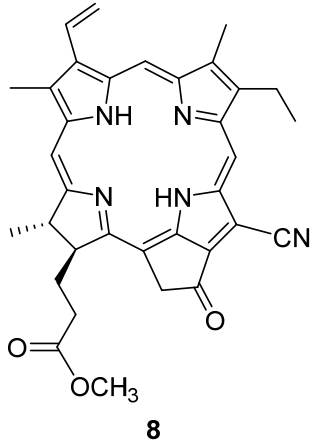

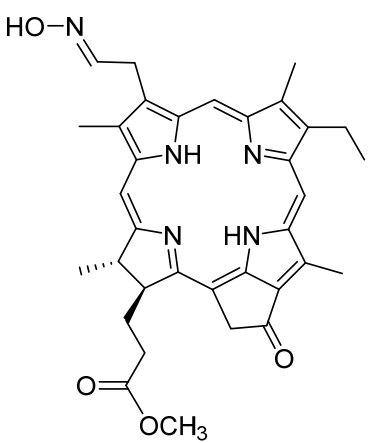

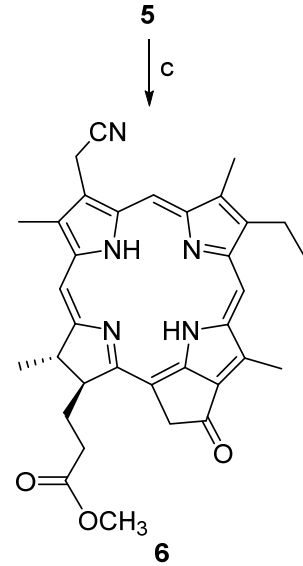

Reagents and conditions: (a) $\mathrm{Tl}\left(\mathrm{NO}_{3}\right)_{3} / \mathrm{CH}_{2} \mathrm{Cl}_{2} / 80 \% \mathrm{HCO}_{2} \mathrm{H}$; (b) $\mathrm{NH}_{2} \mathrm{OH} \mathrm{HCl} / \mathrm{MeOH} / \mathrm{con}$. $\mathrm{HCl}$; (c) DMF/TCT; (d) LiOH/THF/O (e) $\mathrm{NH}_{2} \mathrm{OH} \mathrm{HCl} / \mathrm{NMP} / 120^{\circ} \mathrm{C}$

图式 1 单腈基取代叶绿素 $\mathrm{a}$ 衍生物的合成

Scheme 1 Synthesis of mono-nitrile substituted chlorophyll a derivatives 


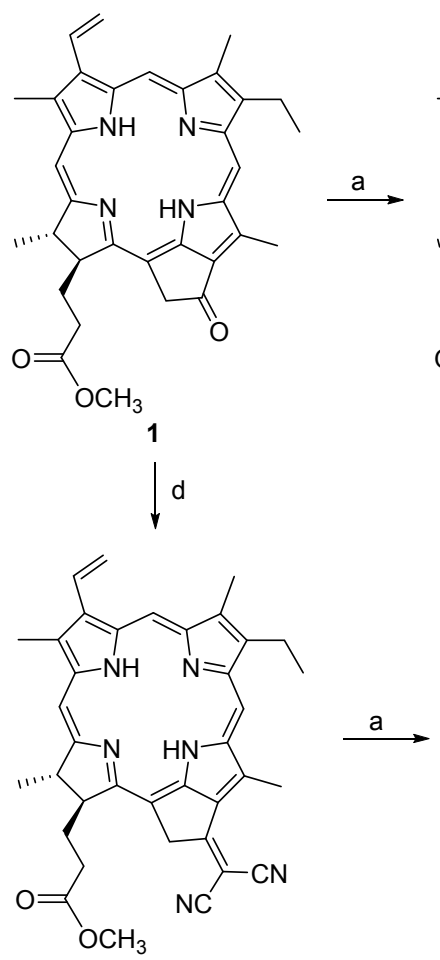

12
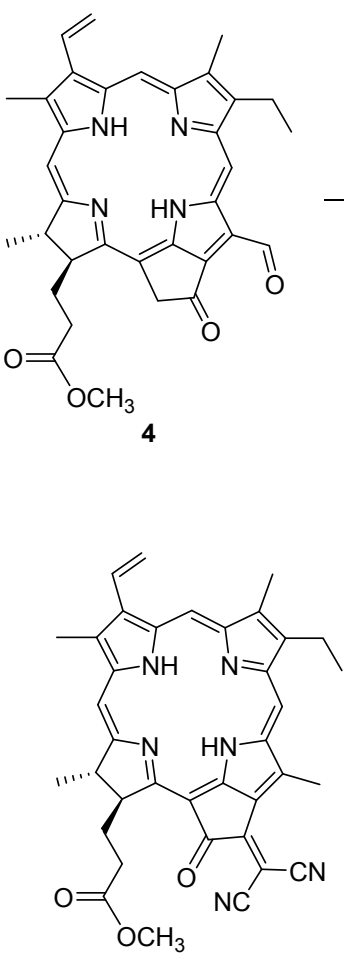

13
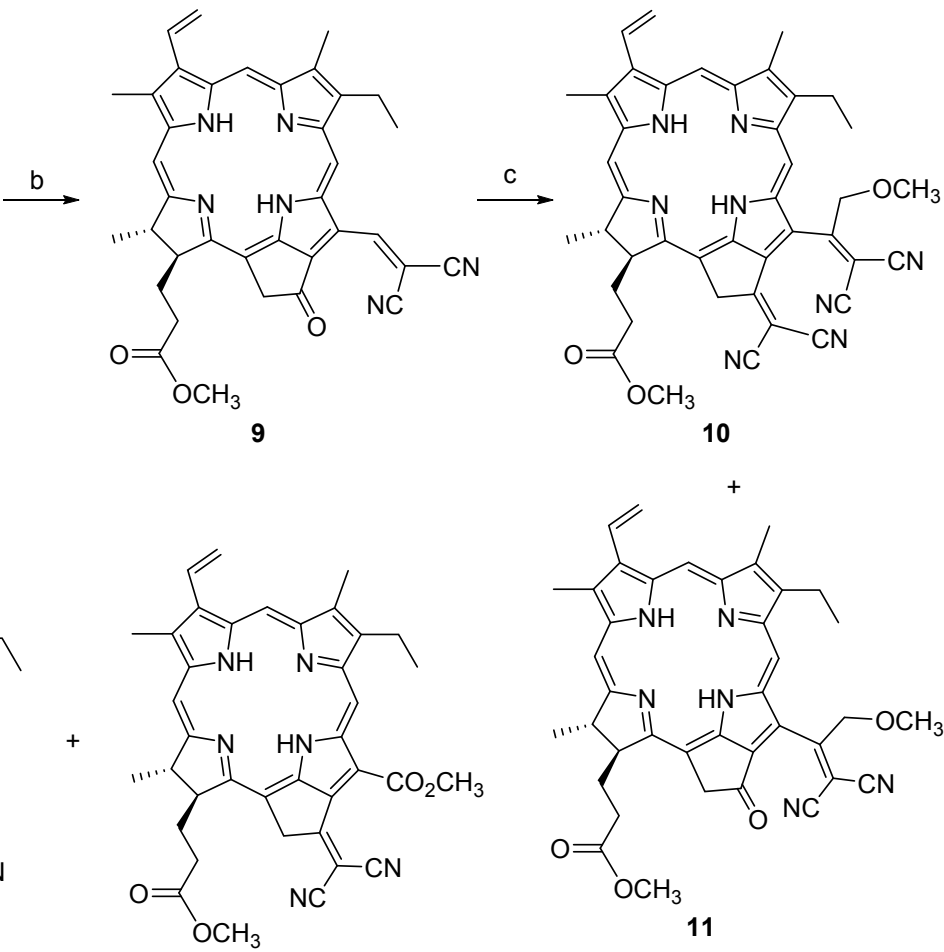

Reagents and conditions: (a) $\mathrm{LiOH} / \mathrm{THF} / \mathrm{O}_{2} / \mathrm{CH}_{2} \mathrm{~N}_{2}$; (b) $\mathrm{TEA} / \mathrm{CH}_{2}(\mathrm{CN})_{2} / \mathrm{CH}_{2} \mathrm{Cl}_{2} ;$ (c) $\left.\mathrm{NaOMe} / \mathrm{MeOH} / \mathrm{CH}{ }_{2}(\mathrm{CN})_{2} ; \mathrm{d}\right) \mathrm{CH}{ }_{2}(\mathrm{CN})_{2} / \mathrm{EtOH} / 80{ }^{\circ} \mathrm{C}$

图式 2 双腈基取代叶绿素 $\mathrm{a}$ 衍生物的合成

Scheme 2 Synthesis of di-nitrile substituted chlorophyll a derivatives

促进的反应条件, 在室温条件下, 对叶绿素降解产物的 醛或酮肜实施重排，以期得到相应的酰胺基或者氰基取 代的新型叶绿素衍生物.

以二氢吓吩肜 5 为例, 三氯三嗪和 DMF 共同促进 肜氰转换的反应过程如图 1 所示: 首先, DMF 与三氯三 嗪发生亲核取代反应生成亚胺盐三嗪醚 $\mathbf{a}$, 所取代的氯 负离子再进攻 $\mathbf{a}$ 中的碳氮双键形成中间体 $\mathbf{b}$, 再经电子 转移裂分成氯代亚胺盐 $\mathbf{c}$ 和三嗪氧负离子 $\mathbf{d}$. 如果遵循 贝克曼的重排过程, 醛肜 $\mathbf{5}$ 与 $\mathbf{c}$ 随即发生亲核加成, 生 成质子化 $O$-取代肜中间体 $\mathbf{A}$, 离去质子后形成 $\mathbf{B}$, 处于 反位的烃基迁移后给出亚胺碳正离子 $\mathbf{C}$, 再与水分子作 用得到贝克曼重排产物 D. 然而, 本反应并未形成大环 色基参与重排的 $\mathrm{C}(3)$-甲酰氨甲基取代的二氢卟吩，而 是得到氢原子发生迁移的 3-氧甲基焦脱镁叶绿酸-a 甲 酯(6). 由于氢迁移时大环色基与重排部分一直保持着 $\pi$-体系共轭, 因而占有较大空间的四吡咯大环没有参与 迁移. 所以，氧基的形成应该是质子化 $O$-取代肟中间体 $\mathbf{A}$ 经历了脱水过程, 即三嗪氧负离子 $\mathbf{d}$ 捕获中间体 $\mathbf{A}$ 中 的 $3 \mathrm{a}$-位质子转化成三嗪 $\mathbf{e}$, 再经电子转移形成碳氮参 键; 与此同时, $O$-取代肟结构中的氮氧键断裂, 离去一 分子 $\mathrm{DMF}$ 和 $\mathrm{HCl}$ 而生成化合物 6 (图 1 框格中所示).

12-甲酰基向氰基转换则起始于二氢卟吩醛 4 在
NMP 中与盐酸差胺的缩合反应, 形成的醛肜 $\mathbf{E}$ 和 $N$-甲 基吡咯酮季铵盐互变异构体 $\mathbf{f}$, 其氢质子迁移至醛肜氧 原子上实施质子化，而氯负离子则俘获质子化醛肜 $\mathbf{F}$ 中 亚胺碳上的质子并促成脱水，分别给出 12-氧基取代的 焦脱镁叶绿酸 8 和 $N$-甲基吡咯烷酮.

\section{2 二氢卟吩中 C-吡咯子环所连酰基的氰基化}

羰基与丙二腈的瑙文格尔缩合可以有效地建立一 个二氰亚甲基结构, 作者也曾利用 3 -位甲酰基和 $E$-环酮 基得到具有特殊光物理性质的二氰基和四氰基取代的 叶绿素衍生物 ${ }^{[9]}$. 本文基于相同的反应, 在 12-位上同样 引进了二氧亚甲基, 并尝试与丙二腈继续反应来进一步 实施氰基化，结果意外地得到四氰基取代和二氰基取代 的二氢卟吩 10 和 11, 其形成过程如图 2 所示: 在含有丙 二腈的甲醇钠/甲醇溶液中, 化合物 9 部分地完成 $E$-环 $13^{1}$-位二氰亚甲基化的同时，12-位上的二氰亚甲基与丙 二腈发生了迈克尔加成, 生成的加成物 $\mathbf{G}$ 脱去一分子氢 氧酸后转化成中间体 $\mathbf{H}$, 再异构成 12 -位上三氰基取代 的更为稳定的中间体 I，最后与甲氧负离子发生亲核取 代反应而分别给出 10 和 11.

为了获取多氰基取代的叶绿素类二氢卟吩，选择氢 氧化锂催化的反应条件, 对化合物 12 实施空气氧化以 期在 $13^{2}$-位和 12 -位上建立新的羰基结构. 与 $\mathrm{MPPa}$ 相 

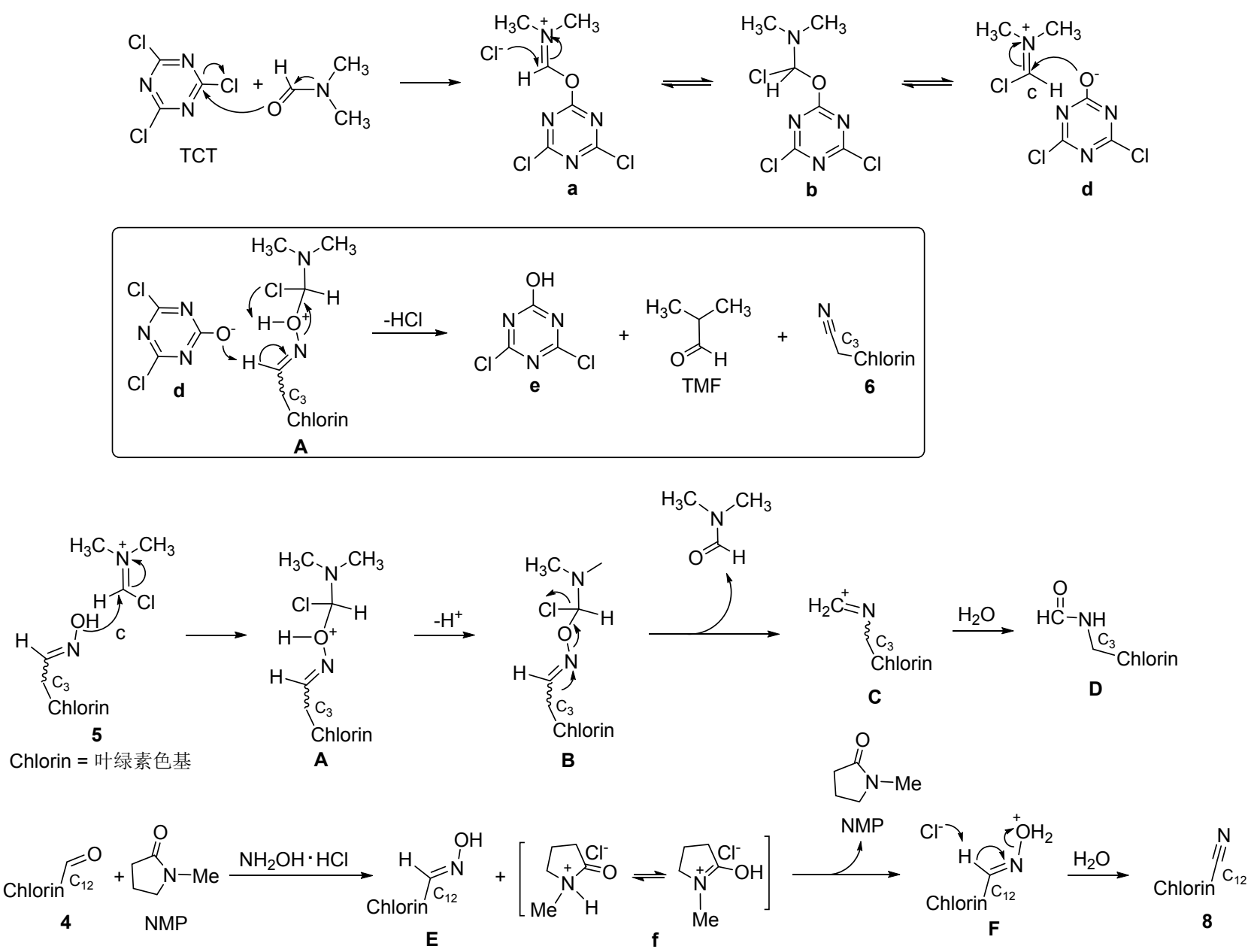

图 1 焦脱镁叶绿酸的周环上醛基向氰基的转化过程

Figure 1 The transformation process of the peripheric formoyl to cyano-group linked with methyl pyropheophorbide

比，化合物 12 的空气氧化需要较长的反应时间，反应结 果也比较混杂, 最后, 从难于分离的混合物中只得到 $13^{2}$-氧代焦脱镁叶绿酸 13 和 12-甲氧甲酰基焦脱镁叶绿 酸 14. 其碳氧双键的形成过程如前文所述 ${ }^{[11]}$, 首先, 在 碱性条件下形成负碳离子 $\mathbf{J}$ 和 $\mathbf{K}$, 再与空气氧分子相互 作用氧化成 13 和二氢卟吩醛 $\mathbf{L}$, 其 12-位甲酰基受到氢 氧负离子的亲核进攻后形成四面体中间体 $\mathbf{M}$, 离去氢 负离子并与体系中的羰基化合物 $\mathbf{N}$ 继续发生反应(类似 于坎尼查罗反应(Cannizzaro reaction), 再经酸化和甲基 化而形成化合物 $\mathbf{1 5}$.

\section{3 氭基化叶绿素类二氢卟吩衍生物的体外光动力活} 性

卟啉类光敏剂的 PDT 抗癌疗效在某种程度上决定 于周环上不同取代基团的空间和电子效应. 考虑到碳氮 参键结构的特殊性, 氰基化对叶绿素类二氢卟吩的光动 力活性势必形成一定的影响, 分别选择 1, 4, 6 12 为待 测化合物. 以中位 meso-四着茎苯基二氢卟吩 (mTHPC)和 临床光动力抗癌药物光敏素(Photofrin)为对照品, 在光
照条件下，采用噻唑蓝(MTT)比色法对上述化合物对小 鼠肉瘤 S-180 细胞的体外光动力活性进行测定. 以所测 化合物浓度的对数为因变量, 以细胞抑制率为应变量作 求得半抑制率 $\left(\mathrm{IC}_{50}\right)$.

从表 1 可以看出, 所有测试的二氢卟吩对小鼠肉瘤 S-180 细胞都具有较强的光动力活性, 其 $\mathrm{IC}_{50}$ 值均低于 $0.600 \mu \mathrm{mol} / \mathrm{L}$ ，对病毒细胞的抑制能力远高于第一代临 床的光动力抗癌药物 Photofrin. 除了化合物 12 以外, 其 他二氢卟吩的 PDT 活性均高于作为叶绿素最初降解产 物的焦脱镁叶绿酸-a 甲酯(1). 尽管大部分测试化合物 的 PDT 活性低于目前临床应用效果最好光敏药物 mTHPC，但 12-位二氰亚甲基焦脱镁叶绿酸 9 的相应活 性与其较为接近，而 15-位连有氰基的二氢卟吩 7 和四 氰基取代的化合物 $\mathbf{1 0}$ 的相应抑制效力甚至高于 mTHPC.

当在周环上引进氰基以后，所得大环分子对小鼠肉 瘤 S-180 细胞的抑制率形成不同的变化趋势. 与 $\mathbf{1}$ 相比, $13^{1}$-二氧亚甲基二氢卟吩 12 的光动力活性有所下降，而 


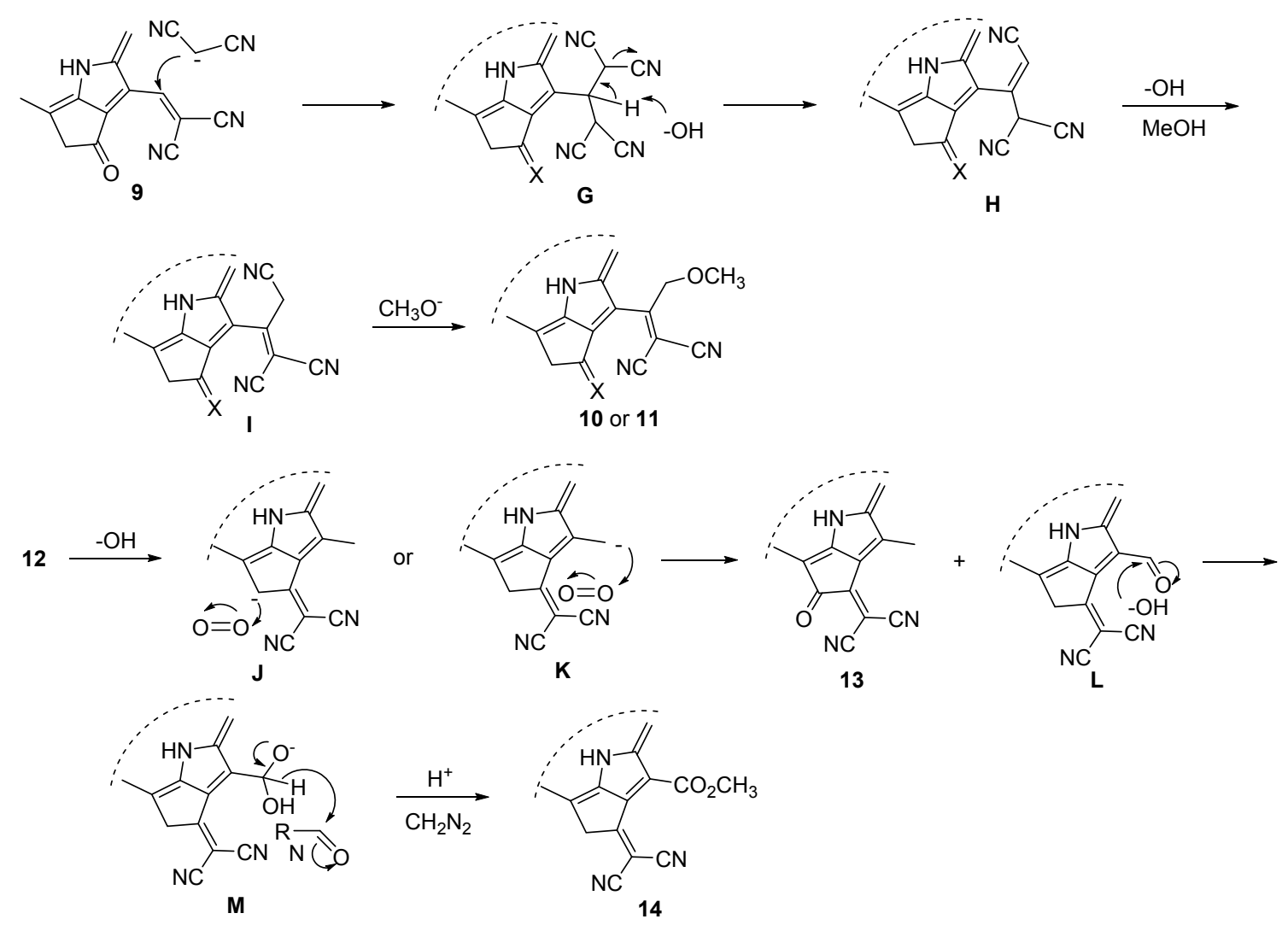

图 2 焦脱镁叶绿酸外接环上和 12-位的氰基化和空气氧化历程

Figure 2 The cyanation and allomerization process on the exocyclic ring and at 12-position of pyropheophorbide

12-位二氰亚甲基叶绿素衍生物对小鼠肉瘤 S-180 细胞 的则表现出较高的抑制力; 结合四氰基二氢卟吩 10 所 具显示出的较高 PDT 活性, 反映出 12-位氰基化比 $13^{2}$ 位氰基化更有益于提高大环分子的 PDT 活性. 参比 $E-$ 环开链结构的 15-㲵基二氢卟吩- $f$ 二甲酯 7 和具有较大 极性的多氰基取代焦脱镁叶绿酸 10 所表现出的较高光 动力活性, 外接环结构保持不变的二氢卟吩 $(1 、 4 、 8$ 和 $12)$ 的半抑制率 $\mathrm{IC}_{50}$ 则相对较低. 测试结果表明, 所连接 的取代基团的极性越高, 其光动力抗癌活性越强. 例如, $13^{1}$-二氯亚甲基焦脱镁叶绿酸 12 的 $\mathrm{IC}_{50}$ 值为 0.596 $\mu \mathrm{mol} / \mathrm{L}$, 而具有更大极性的氧化同系物 $\mathbf{1 0}$ 的相应测定 值则降至 $0.048 \mu \mathrm{mol} / \mathrm{L}$; 二氢卟吩醛 4 的极性要高于其 二氰亚甲基化产物 $\mathbf{8}$, 前者的 PDT 肿瘤抑制活性也比后 者要高.

\section{2 结论}

利用不同氧化剂氧化和空气氧化反应, 在叶绿素降
解产物周环的不同位置上引进甲酰基团，与盐酸着胺肜 化后，在不同的反应条件下可以顺利地将环上醛基转化 为氰基; 通过 $\mathrm{C}$-吡咯子环所连带的碳氧双键与丙二腈 的瑙文格尔缩合，在 12-位和五元外接环上建立了二氰 亚甲基结构, 其进一步的结构转换能够形成新的多氰基 取代的四吡咯大环产物. 氧基结构的引入对大环分子的 光动力活性能够产生明显的影响, 同时, 利用所连带氰 基的结构转换, 为获取新型叶绿素类二氢卟吩衍生物提 供了更加广泛的合成途径.

\section{3 实验部分}

\section{1 仪器与试剂}

元素分析用 Perkin-Elmer 2400 型元素分析仪测定; IR 用 Perkin-Elmer 1730 型红外分光光度仪测定 $(K B r$ 压 片); UV-Vis 用 UV-160A 型紫外分光光度计测定; ${ }^{1} \mathrm{H}$ NMR 用 Brucker ARX-400 型核磁共振仪测定, 内标 为 TMS. 所用试剂均为分析纯或化学纯. 焦脱镁叶绿

表 1 叶绿素类二氢卟吩对小鼠肉瘤 -180 细胞的半抑制率 $\left(\mathrm{IC}_{50}\right)$

Table $1 \mathrm{IC}_{50}$ results of chlorophyllous chlorins on mouse sarcoma S-180 cell line

\begin{tabular}{lccccccccccc}
\hline Comd. & Photofin & mTHPD & $\mathbf{1}$ & $\mathbf{4}$ & $\mathbf{6}$ & $\mathbf{7}$ & $\mathbf{8}$ & $\mathbf{9}$ & $\mathbf{1 0}$ & $\mathbf{1 1}$ & $\mathbf{1 2}$ \\
\hline $\mathrm{IC}_{50} /\left(\mu \mathrm{mol} \cdot \mathrm{L}^{-1}\right)^{a}$ & 0.776 & 0.061 & 0.389 & 0.239 & 0.187 & 0.052 & 0.328 & 0.072 & 0.048 & 0.247 & 0.596 \\
\hline
\end{tabular}

a 所测光敏剂及对照在只加光敏剂无光照以及只光照无光敏剂条件下对小鼠肉瘤 -180 细胞的半抑制率 $\left(\mathrm{IC}_{50}\right)$ 均大于所测最高浓度 $\left(10 \mu \mathrm{mol} \cdot \mathrm{L}^{-1}\right)$. 
酸-a 甲酯(1)按文献[12]制备.

\subsection{5-甲酰基二氢卟吩- $f$ 二酯(3)和 12-甲酰基焦脱镁 叶绿酸-a 甲酯(4)的合成}

将 $536 \mathrm{mg}$ (0.978 mmol)焦脱镁叶绿酸甲酯-a (1)溶 解于 $280 \mathrm{~mL}$ 四氢呋喃中, 再加入由氢氧化锂饱和的 15 $\mathrm{mL}$ 甲醇溶液, 室温搅拌 $3 \mathrm{~h}$; 加入 $200 \mathrm{~mL}$ 冰水, 搅拌下 滴加浓盐酸, 调节 $\mathrm{pH}$ 为 $2 \sim 3$, 加入 $200 \mathrm{~mL}$ 二氯甲烷分 层, 用二氯甲烷萃取水相 $(40 \mathrm{~mL} \times 2)$, 合并有机层, 水 洗, 用无水硫酸钠干燥, 蒸馏除去溶剂. 然后重新溶解 于 $350 \mathrm{~mL}$ 含有 $5 \%$ 浓硫酸的甲醇溶液, 室温搅拌过夜. 先后加入 $300 \mathrm{~mL}$ 水和 $300 \mathrm{~mL}$ 二氯甲烷分层, 分出有机 相并水洗两次, 干燥后除去溶剂, 将剩余物通过柱层析 分离 [洗脱剂: $V($ 乙酸乙酯 $): V($ 正己烷 $)=1: 4]$ 从较大极 性色带中分离出 $46 \mathrm{mg}$ 黄色固体 $3(0.078 \mathrm{mmol}, 8 \%)$ 和 $104 \mathrm{mg}$ 绿色固体 4 (0.186 mmol, 19\%). 化合物 3 和 4 的 分析数据和物理常数与文献 $[11]$ 一致.

\section{3 (E/Z)-3-羟胺亚乙基-3-去乙烯基焦脱镁叶绿酸-a 甲酯(5)的合成}

在 $50 \mathrm{~mL}$ 二氯甲烷和 $25 \mathrm{~mL}$ 甲醇混合液中溶解 198 mg MPPa 1 (0.361 mmol), 在 $0{ }^{\circ} \mathrm{C}$ 下将溶解于 $30 \mathrm{~mL}$ 甲 醇的 $150 \mathrm{mg}$ 三水硝酸铊迅速加入到反应液中, 并在此 温度下搅拌反应 $15 \mathrm{~min}$, 然后加入 $30 \mathrm{~mL}$ 亚硫酸氢钠甲 醇饱和溶液, 室温下搅拌 $20 \mathrm{~min}$, 滴加 $2 \mathrm{~mL}$ 浓盐酸酸 化, 将大量的白色沉淀过滤, 滤液用二氯甲烷萃取 (20 $\mathrm{mL} \times 3)$, 分出有机层水洗, 干燥后减压浓缩至干, 然后 重新溶解于 $15 \mathrm{~mL} 80 \%$ 的甲酸溶液中, 室温摚拌 $6 \mathrm{~h}$, 加 入 $20 \mathrm{~mL}$ 水和 $35 \mathrm{~mL}$ 二氯甲烷, 分出有机层, 水层用二 氯甲烷萃取 $(15 \mathrm{~mL} \times 3)$, 合并有机层并用水洗涤, 无水 硫酸钠干燥, 减压浓缩, 硅胶柱层析分离 [洗脱剂: $V$ (石油醚) $: V($ 乙酸乙酯 $)=3 ： 1]$, 得到 $148 \mathrm{mg}$ 暗红色 固体 $2(0.260 \mathrm{mmol}, 72 \%)$. 将所得固体溶解于 $10 \mathrm{~mL}$ 甲醇中, 再加入溶有 $40 \mathrm{mg}$ 盐酸着弪胺的 $10 \mathrm{~mL}$ 甲醇, 搅拌下滴加 $0.1 \mathrm{~mL}$ 浓盐酸, 常温搅拌反应 $1 \mathrm{~h}$, 先后 加入 $20 \mathrm{~mL}$ 二氯甲烷和 $20 \mathrm{~mL}$ 水分层, 水相用二氯甲烷 萃取(15 mL×3), 合并有机层, 水洗后用无水硫酸钠干 燥, 减压除去溶剂, 剩余物经硅胶柱层析[洗脱剂: $V($ 石 油醚) : $V$ (乙酸乙酯) $=2: 1$ ], 得到 $53 \mathrm{mg}$ 黑绿色固体 5, 产率 $42 \%$. m.p. $188 \sim 191{ }^{\circ} \mathrm{C}$; UV-vis $\left(\mathrm{CH}_{2} \mathrm{Cl}_{2}\right) \lambda_{\max }$ $\left[\varepsilon /\left(\mathrm{L} \cdot \mathrm{mol}^{-1} \cdot \mathrm{cm}^{-1}\right)\right]: 408\left(1.87 \times 10^{4}\right), 503\left(1.50 \times 10^{3}\right)$, $533\left(1.31 \times 10^{3}\right), 603\left(1.12 \times 10^{3}\right), 660\left(7.11 \times 10^{3}\right) \mathrm{nm} ;{ }^{1} \mathrm{H}$ NMR $\left(\mathrm{CDCl}_{3}\right) \delta$ : -1.11 (br s, $\left.1 \mathrm{H}, \mathrm{NH}\right), 0.65$ (br s, $1 \mathrm{H}$, $\mathrm{NH}), 1.59$ (t, $\left.J=7.6 \mathrm{~Hz}, 3 \mathrm{H}, 8-\mathrm{CH}_{3}\right), 1.79(\mathrm{~d}, J=7.2 \mathrm{~Hz}$, $\left.3 \mathrm{H}, 18-\mathrm{CH}_{3}\right), 1.98 \sim 2.08(\mathrm{~m}, 1 \mathrm{H}, 17 \mathrm{a}+17 \mathrm{~b}-\mathrm{H}), 2.23 \sim$ $2.35(\mathrm{~m}, 1 \mathrm{H}, 17 \mathrm{a}+17 \mathrm{~b}-\mathrm{H}), 2.42 \sim 2.60(\mathrm{~m}, 2 \mathrm{H}, 17 \mathrm{a}+$ 17b-H), 2.97, 3.19, 3.28, 3.66 (each s, each $3 \mathrm{H}, \mathrm{CH}_{3}+$
$\left.\mathrm{OCH}_{3}\right), 3.57$ (q, $\left.J=7.6 \mathrm{~Hz}, 2 \mathrm{H}, 8 \mathrm{a}-\mathrm{H}\right), 4.05$ (d, $J=9.4 \mathrm{~Hz}$, $1 \mathrm{H}, 17-\mathrm{H}), 4.34$ (q, $J=7.2 \mathrm{~Hz}, 1 \mathrm{H}, 18-\mathrm{H}), 4.83$ (d, $J=1.5$ $\mathrm{Hz}, 2 \mathrm{H}, 3 \mathrm{a}-\mathrm{H}), 4.99$ (d, $\left.J=20.8 \mathrm{~Hz}, 1 \mathrm{H}, 13^{2}-\mathrm{H}\right), 5.15$ (d, $\left.J=20.8 \mathrm{~Hz}, 1 \mathrm{H}, 13^{2}-\mathrm{H}\right), 8.41(\mathrm{~s}, 1 \mathrm{H}, 3 \mathrm{~b}-\mathrm{H}), 8.85,8.95$, 10.15 (each s, each $1 \mathrm{H}$, meso-H); ${ }^{13} \mathrm{C}$ NMR $(400 \mathrm{~Hz}$, $\left.\mathrm{CDCl}_{3}\right) \delta: 195.86,173.43,170.28,169.00,161.68,154.89$, $152.11,150.92,150.87,148.65,144.65,138.82,136.58$, $133.27,131.34,125.00,106.66,103.55,99.08,93.83$, $99.83,93.89,52.00,51.75,49.67,48.11,31.00,29.79$, $23.24,19.91,19.23,17.28,11.96,10.84$; IR (KBr) v: 3324 $(\mathrm{N}-\mathrm{H}), 2925(\mathrm{C}-\mathrm{H}), 1739,1696(\mathrm{C}=\mathrm{O}), 1602(\mathrm{C}=\mathrm{C})$, 1500 (chlorin skeleton), 1451, 1263, 1089, 1041, 903 $\mathrm{cm}^{-1}$; EI-MS m/z: $580.4\left(\mathrm{M}+\mathrm{H}^{+}\right)$. Anal. calcd for $\mathrm{C}_{34} \mathrm{H}_{37} \mathrm{~N}_{5} \mathrm{O}_{4}$ : C 70.45, H 6.43, N 12.08; found $\mathrm{C} 70.39, \mathrm{H}$ 6.32, N 12.20.

\subsection{3-氭甲基-3-去乙烯基焦脱镁叶绿酸-a 甲酯(6)的合 成}

在 $2 \mathrm{~mL}$ DMF 中溶解 $480 \mathrm{mg}$ 三氯三嗪, 室温搅拌 $30 \mathrm{~min}$ (体系的颜色由无色逐渐变为乳红色), 然后与含 有 $133 \mathrm{mg}$ 二氢叶吩醛肟 $5(0.230 \mathrm{mmol})$ 的 $5 \mathrm{~mL} \mathrm{DMF}$ 合 并, 室温搅拌 $24 \mathrm{~h}$. 先后加入 $15 \mathrm{~mL}$ 水和 $25 \mathrm{~mL}$ 二氯甲 烷促使反应体系分层, 分出有机相, 水相用二氯甲烷萃 取(15 mL×3), 合并有机相并水洗, 无水硫酸钠干燥, 浓缩后用硅胶柱层析分离[洗脱剂: $V$ (石油醚)： $V$ (乙酸 乙酯 $)=4: 1$ ], 得 $62 \mathrm{mg}$ 黑色固体 $6(0.106 \mathrm{mmol})$, 产率 $48 \%$. m.p. $188 \sim 191{ }^{\circ} \mathrm{C}$, UV-vis $\left(\mathrm{CH}_{2} \mathrm{Cl}_{2}\right) \lambda_{\max }[\varepsilon /(\mathrm{L} \bullet$ $\left.\left.\mathrm{mol}^{-1} \cdot \mathrm{cm}^{-1}\right)\right]: 316\left(1.84 \times 10^{3}\right), 406\left(1.53 \times 10^{4}\right), 504$ $\left(9.18 \times 10^{2}\right), \quad 534 \quad\left(8.35 \times 10^{2}\right), \quad 608 \quad\left(7.65 \times 10^{2}\right), \quad 664$ $\left(1.19 \times 10^{4}\right) \mathrm{nm} ;{ }^{1} \mathrm{H}$ NMR $\left(\mathrm{CDCl}_{3}\right) \delta:-1.88($ br s, $1 \mathrm{H}$, $\mathrm{NH}), 0.16$ (br s, 1H, NH), 1.69 (t, $J=7.6 \mathrm{~Hz}, 3 \mathrm{H}, 8-\mathrm{CH}_{3}$ ), $1.81\left(\mathrm{~d}, J=7.2 \mathrm{~Hz}, 3 \mathrm{H}, 18-\mathrm{CH}_{3}\right), 2.25 \sim 2.33(\mathrm{~m}, 1 \mathrm{H}$, $17 \mathrm{a}+17 \mathrm{~b}-\mathrm{H}), 2.53 \sim 2.61(\mathrm{~m}, 2 \mathrm{H}, 17 \mathrm{a}+17 \mathrm{~b}-\mathrm{H}), 2.66 \sim$ $2.74(\mathrm{~m}, 1 \mathrm{H}, 17 \mathrm{a}+17 \mathrm{~b}-\mathrm{H}), 3.25,3.39,3.62,3.65$ (each $\mathrm{s}$, each $3 \mathrm{H}, \mathrm{CH}_{3}+\mathrm{OCH}_{3}$ ), 3.66 (q, $\left.J=7.6 \mathrm{~Hz}, 2 \mathrm{H}, 8 \mathrm{a}-\mathrm{H}\right)$, $4.31(\mathrm{~d}, J=8.6 \mathrm{~Hz}, 1 \mathrm{H}, 17-\mathrm{H}), 4.53$ (q, $J=7.2 \mathrm{~Hz}, 1 \mathrm{H}$, $18-\mathrm{H}), 4.81$ (s, 2H, 3a-H), 5.12 (d, $\left.J=19.8 \mathrm{~Hz}, 1 \mathrm{H}, 13^{2}-\mathrm{H}\right)$, 5.27 (d, $\left.J=19.8 \mathrm{~Hz}, 1 \mathrm{H}, 13^{2}-\mathrm{H}\right), 8.59,9.20,9.51$ (each s, each $1 \mathrm{H}$, meso-H); ${ }^{13} \mathrm{C} \mathrm{NMR}\left(400 \mathrm{~Hz}, \mathrm{CDCl}_{3}\right) \delta: 200.09$, $173.61,170.95,160.83,155.21,150.58,148.565,145.07$, $141.45,138.10,136.38,136.09,135.88,131.72,130.59$, $129.31,129.22,122.63,112.32,104.15,93.13,53.31$, 50.99, 31.88, 31.78, 29.86, 23.50, 19.58, 17.92, 17.54, 12.23, 11.68; IR (KBr) v: $3445(\mathrm{~N}-\mathrm{H}), 2958,2835(\mathrm{C}-$ $\mathrm{H}), 1736,1689(\mathrm{C}=\mathrm{O}), 1655(\mathrm{C}=\mathrm{C}), 1527$ (chlorin skeleton), 1400, 1286, 1189, $1094 \mathrm{~cm}^{-1}$; EI-MS m/z: 561.7 
$\left(\mathrm{M}+\mathrm{H}^{+}\right)$. Anal. calcd for $\mathrm{C}_{34} \mathrm{H}_{35} \mathrm{~N}_{5} \mathrm{O}_{3}: \mathrm{C} 72.71, \mathrm{H} 6.28, \mathrm{~N}$ 12.47; found C 72.59, H 6.32, N 12.30 .

\subsection{5-氰基二氢卟吩 $f$ 二甲酯(7)的合成}

在 $10 \mathrm{~mL}$ 甲醇中溶解 $70 \mathrm{mg}$ 二氢卟吩醛 $3(0.118$ $\mathrm{mmol}$ ), 搅拌下加入研成粉末的 $40 \mathrm{mg}$ 盐酸羟胺, 继 续摚拌 $5 \mathrm{~min}$, 滴加 $0.1 \mathrm{~mL}$ 浓盐酸后常温摚拌反应 1 $\mathrm{h}$; 先后加入 $20 \mathrm{~mL}$ 二氯甲烷和 $20 \mathrm{~mL}$ 水, 分出有机相 并水洗两次, 减压除去溶剂, 剩余物重新溶解于 $5 \mathrm{~mL}$ DMF 中, 然后倒入溶有 $480 \mathrm{mg}$ 三氯三嗪的 $2 \mathrm{~mL}$ DMF 中, 室温摚拌 $24 \mathrm{~h}$. 先后加入 $15 \mathrm{~mL}$ 水和 $25 \mathrm{~mL}$ 二氯甲 烷促使反应体系分层, 分出有机层, 水层用二氯甲烷萃 取(15 $\mathrm{mL} \times 3)$, 合并有机相并水洗, 无水硫酸钠干燥, 浓缩后用硅胶柱层析分离 [洗脱剂: $V$ (石油醚) : $V$ (乙酸 乙酯 $)=4: 1$ ], 得 $18 \mathrm{mg}$ 黑色固体 $7(0.031 \mathrm{mmol})$, 产率 26\%. m.p. $191 \sim 194{ }^{\circ} \mathrm{C}$; UV-Vis $\left(\mathrm{CH}_{2} \mathrm{Cl}_{2}\right) \lambda_{\max }[\varepsilon /(\mathrm{L} \bullet$ $\left.\left.\mathrm{mol}^{-1} \cdot \mathrm{cm}^{-1}\right)\right]$ ]: $385\left(5.23 \times 10^{3}\right), 422\left(1.07 \times 10^{4}\right), 512$ $\left(9.42 \times 10^{2}\right), 561\left(5.01 \times 10^{3}\right), 688\left(9.34 \times 10^{3}\right) \mathrm{nm} ;{ }^{1} \mathrm{H}$ NMR $\left(\mathrm{CDCl}_{3}\right) \delta:-1.15$ (br s, $\left.2 \mathrm{H}, \mathrm{NH}\right), 1.65$ (t, $J=7.6$ $\left.\mathrm{Hz}, 3 \mathrm{H}, 8 \mathrm{a}-\mathrm{CH}_{3}\right), 1.79\left(\mathrm{~d}, J=7.2 \mathrm{~Hz}, 3 \mathrm{H}, 18-\mathrm{CH}_{3}\right), 2.24 \sim$ $2.32(\mathrm{~m}, 1 \mathrm{H}, 17 \mathrm{a}+17 \mathrm{~b}-\mathrm{H}), 2.53 \sim 2.62(\mathrm{~m}, 2 \mathrm{H}, 17 \mathrm{a}+$ 17b-H), $2.67 \sim 2.75$ (m, 1H, 17a+17b-H), 3.18, 3.40, 3.57, $3.59,4.37$ (each s, each $3 \mathrm{H}, \mathrm{CH}_{3}+\mathrm{OCH}_{3}$ ), 3.64 (q, $J=7.6$ $\mathrm{Hz}, 2 \mathrm{H}, 8 \mathrm{a}-\mathrm{H}), 4.51$ (q, $J=7.2 \mathrm{~Hz}, 1 \mathrm{H}, 18-\mathrm{H}), 4.75$ (dd, $J=9.0,3.0 \mathrm{~Hz}, 1 \mathrm{H}, 17-\mathrm{H}), 6.14(\mathrm{~d}, J=11.6 \mathrm{~Hz}, 1 \mathrm{H}$, cis-3b-H), 6.28 (d, $J=17.8 \mathrm{~Hz}, 1 \mathrm{H}$, trans-3b-H), 7.94(dd, $J=17.8,11.6 \mathrm{~Hz}, 3 \mathrm{a}-\mathrm{H}), 8.71,9.52,9.63\left(\right.$ each s, $2 \mathrm{H}$, me- $^{-}$ so-H); IR(KBr) v: $33419(\mathrm{~N}-\mathrm{H}), 2915,2847(\mathrm{C}-\mathrm{H})$, 1733, $1691(\mathrm{C}=\mathrm{O}), 1604(\mathrm{C}=\mathrm{C}), 1497$ (chlorin skeleton), 1437, 1365, 1199, 1111, $981 \mathrm{~cm}^{-1}$; EI-MS m/z: $592.3(\mathrm{M}+$ $\mathrm{H}^{+}$). Anal. calcd for $\mathrm{C}_{35} \mathrm{H}_{37} \mathrm{~N}_{5} \mathrm{O}_{4}: \mathrm{C}$ 71.05, H 6.30, N 11.84; found C 71.21, H 6.19, N 11.69.

\subsection{2-氭基焦脱镁叶绿酸-a 甲酯(8)的合成}

在 $8 \mathrm{~mL} \mathrm{~N}$-甲基吡咯烷酮中溶解 $179 \mathrm{mg}$ 二氢卟吩醛 $4(0.319 \mathrm{mmol})$, 搅拌下加入预先研细的 $51 \mathrm{mg}$ 盐酸羟胺 (0.739 mmol), $120{ }^{\circ} \mathrm{C}$ 下搅拌 $8 \mathrm{~h}$. 先后加入 $15 \mathrm{~mL}$ 水和 $25 \mathrm{~mL}$ 二氯甲烷促使反应体系分层, 分出有机相, 水相 用二氯甲烷萃取 $(15 \mathrm{~mL} \times 3)$, 合并有机相并水洗, 无水 硫酸钠干燥, 浓缩后用硅胶柱层析分离 [洗脱剂: $V$ (石油 醚)：V(乙酸乙酯 $)=4: 1$ ], 得 $52 \mathrm{mg}$ 黑色固体 $6(0.093$ mmol), 产率 29\%. m.p. $188 \sim 191{ }^{\circ} \mathrm{C}$; UV-Vis $\left(\mathrm{CH}_{2} \mathrm{Cl}_{2}\right)$

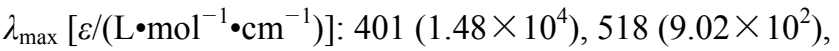
$564\left(8.66 \times 10^{2}\right), 614\left(8.68 \times 10^{2}\right), 670\left(1.32 \times 10^{4}\right) \mathrm{nm} ;{ }^{1} \mathrm{H}$ NMR $\left(\mathrm{CDCl}_{3}\right) \delta: \quad-0.49($ br s, $1 \mathrm{H}, \mathrm{NH}), 0.72($ br s, $1 \mathrm{H}$, $\mathrm{NH}), 1.43$ (t, $\left.J=7.6 \mathrm{~Hz}, 3 \mathrm{H}, 8-\mathrm{CH}_{3}\right), 1.81(\mathrm{~d}, J=7.2 \mathrm{~Hz}$, $\left.3 \mathrm{H}, 18-\mathrm{CH}_{3}\right), 2.13 \sim 2.23(\mathrm{~m}, 1 \mathrm{H}, 17 \mathrm{a}+17 \mathrm{~b}-\mathrm{H}), 2.33 \sim$ $2.43(\mathrm{~m}, 2 \mathrm{H}, 17 \mathrm{a}+17 \mathrm{~b}-\mathrm{H}), 2.54 \sim 2.68(\mathrm{~m}, 1 \mathrm{H}, 17 \mathrm{a}+$ 17b-H), 2.80, 3.25, 3.66 (each s, each $3 \mathrm{H}, \mathrm{CH}_{3}+\mathrm{OCH}_{3}$ ), 3.16 (q, $J=7.6 \mathrm{~Hz}, 2 \mathrm{H}, 8 \mathrm{a}-\mathrm{H}), 4.03(\mathrm{td}, J=9.4,2.0 \mathrm{~Hz}$, $1 \mathrm{H}, 17-\mathrm{H}), 4.29$ (dq, $J=7.4,2.0 \mathrm{~Hz}, 1 \mathrm{H}, 18-\mathrm{H}), 4.85$ (d, $\left.J=19.8 \mathrm{~Hz}, 1 \mathrm{H}, 13^{2}-\mathrm{H}\right), 5.01\left(\mathrm{~d}, J=19.8 \mathrm{~Hz}, 1 \mathrm{H}, 13^{2}-\mathrm{H}\right)$, 6.15 (d, $J=11.6 \mathrm{~Hz}, 1 \mathrm{H}, c i s-3 \mathrm{~b}-\mathrm{H}), 6.19$ (d, $J=17.8 \mathrm{~Hz}$, $1 \mathrm{H}$, trans-3b-H), $7.70(\mathrm{dd}, J=17.8,11.6 \mathrm{~Hz}, 1 \mathrm{H}, 3 \mathrm{a}-\mathrm{H})$, 8.18, 8.51, 8.63 (each s, each $1 \mathrm{H}$, meso-H); ${ }^{13} \mathrm{C}$ NMR (400 $\left.\mathrm{Hz}, \mathrm{CDCl}_{3}\right) \delta: 187.07,173.45,170.94,150.82,149.68$, $144.92,141.16,137.38,136.39,135.73,131.36,129.19$, $128.97,128.93,122.64,112.90,106.88,104.43,99.83$, $93.89,51.65,51.59,31.92,31.23,29.64,29.35,23.59$, 22.68, 19.47, 17.44, 14.10, 12.21, 12.11, 11.22; IR (KBr) $v: 3449(\mathrm{~N}-\mathrm{H}), 2924(\mathrm{C}-\mathrm{H}), 1742,1708(\mathrm{C}=\mathrm{O}), 1686$ $(\mathrm{C}=\mathrm{C}), 1560$ (chlorin skeleton), 1457, 1341, 1082, 1040, $971 \mathrm{~cm}^{-1}$; EI-MS m/z: $560.3\left(\mathrm{M}+\mathrm{H}^{+}\right)$. Anal. calcd for $\mathrm{C}_{34} \mathrm{H}_{33} \mathrm{~N}_{5} \mathrm{O}_{3}$ : C 72.97, H 5.94, N 12.51; found C 72.82, H 6.02, N 12.37 .

\subsection{2-二氭亚甲基-12-去甲基焦脱镁叶绿酸-a 甲酯 (9)的合成}

将 $80 \mathrm{mg}$ 12-甲酰基焦脱镁叶绿酸-a 甲酯(4) (0.142 $\mathrm{mmol}$ )溶解于 $10 \mathrm{~mL}$ 四氢呋喃中, 加入 $0.3 \mathrm{~mL}$ 三乙胺后, 再慢慢滴加溶解于 $5 \mathrm{~mL}$ 四氢呋喃的 $40 \mathrm{mg}$ 丙二睛, 5 $\min$ 内加完, 避光条件下室温摚拌, TLC 监测反应进程, $20 \mathrm{~min}$ 反应结束, 向反应体系加入 $20 \mathrm{~mL}$ 水和 $20 \mathrm{~mL}$ 二 氯甲烷，分出有机层，水层用二氯甲烷萃取 $(15 \mathrm{~mL} \times 3)$, 合并有机层，水洗后用无水硫酸钠干燥，减压浓缩，剩 余物经硅胶柱层析分离 [洗脱剂: $V$ (石油醚) $: V$ (乙酸乙 酯 $)=4: 1$ ], 得到 $65 \mathrm{mg}$ 绿色固体 $9(0.107 \mathrm{mmol})$. 产率 75\%. m.p. $241 \sim 242{ }^{\circ} \mathrm{C}$; UV-Vis $\left(\mathrm{CH}_{2} \mathrm{Cl}_{2}\right) \lambda_{\max }[\varepsilon /(\mathrm{L} \cdot$ $\left.\left.\mathrm{mol}^{-1} \cdot \mathrm{cm}^{-1}\right)\right]: \quad 401\left(9.91 \times 10^{4}\right), 504\left(7.93 \times 10^{3}\right), 624$ $\left(8.92 \times 10^{4}\right), 658\left(1.19 \times 10^{4}\right), 699\left(2.48 \times 10^{4}\right) \mathrm{nm} ;{ }^{1} \mathrm{H}$ NMR $\left(\mathrm{CDCl}_{3}\right) \delta: 0.09$ (br s, 1H, NH), 0.68 (br s, 1H, NH), $1.48\left(\mathrm{t}, J=7.6 \mathrm{~Hz}, 3 \mathrm{H}, 8-\mathrm{CH}_{3}\right), 1.78(\mathrm{~d}, J=7.2 \mathrm{~Hz}, 3 \mathrm{H}$, $\left.18-\mathrm{CH}_{3}\right), 2.11 \sim 2.21(\mathrm{~m}, 1 \mathrm{H}, 17 \mathrm{a}+17 \mathrm{~b}-\mathrm{H}), 2.28 \sim 2.40$ (m, 2H, 17a+17b-H), 2.51 2.62 (m, 1H, 17a+17b-H), 2.94, 3.23, 3.66 (each s, each $3 \mathrm{H}, \mathrm{CH}_{3}+\mathrm{OCH}_{3}$ ), 4.03 (q, $J=7.6 \mathrm{~Hz}, 2 \mathrm{H}, 8 \mathrm{a}-\mathrm{H}), 4.23(\mathrm{q}, J=7.2 \mathrm{~Hz}, 1 \mathrm{H}, 18-\mathrm{H}), 4.05$ (d, $J=8.4 \mathrm{~Hz}, 1 \mathrm{H}, 17-\mathrm{H}), 4.90\left(\mathrm{~d}, J=20.0 \mathrm{~Hz}, 1 \mathrm{H}, 13^{2}-\mathrm{H}\right)$, $5.05\left(\mathrm{~d}, J=20.0 \mathrm{~Hz}, 1 \mathrm{H}, 13^{2}-\mathrm{H}\right), 6.15(\mathrm{~d}, J=11.6 \mathrm{~Hz}, 1 \mathrm{H}$, cis-3b-H), 6.20 (d, J=17.8 Hz, 1H, trans-3b-H), 7.72 (dd, $J=17.8,11.6 \mathrm{~Hz}, 1 \mathrm{H}, 3 \mathrm{a}-\mathrm{H}), 8.53$ (s, 1H, 12-H), 8.21, 8.64, 8.69 (each s, each $1 \mathrm{H}$, meso-H); IR (KBr) v: 3443 $(\mathrm{N}-\mathrm{H}), 2957(\mathrm{C}-\mathrm{H}), 1731,1698(\mathrm{C}=\mathrm{O}), 1623(\mathrm{C}=\mathrm{C})$, 1560 (chlorin skeleton), 1462, 1232, 1170, 1031, 916 
$\mathrm{cm}^{-1}$; EI-MS $m / z$ : $611.3\left(\mathrm{M}+\mathrm{H}^{+}\right)$. Anal. calcd for $\mathrm{C}_{37} \mathrm{H}_{34} \mathrm{~N}_{6} \mathrm{O}_{3}$ : C 72.77, H 5.61, N 13.76; found C 72.81, H $5.51, \mathrm{~N} 13.59$.

$3.813^{2}$-二氭亚甲基-12-( 1 '-甲氧甲基- $2^{\prime}, 2^{\prime}$-二氭基乙 烯基)-132-去氧-12-去甲基焦脱镁叶绿酸-a 甲酯(10)和 12-(1'-甲氧甲基-2', 2'-二氭基乙烯基)-12-去甲基焦脱镁 叶绿酸-a 甲酯(11)的合成

将 $129 \mathrm{mg} 12-\beta, \beta$-二氧亚甲基焦脱镁叶绿酸(9) $(0.211 \mathrm{mmol})$ 溶解于 $15 \mathrm{~mL}$ 含有 $20 \mathrm{mg}$ 甲醇钠的甲醇中, 搅拌下加入 $130 \mathrm{mg}$ 丙二腈 $(0.002 \mathrm{~mol})$, 于 $35{ }^{\circ} \mathrm{C}$ 氮气保 护下避光搅拌, $3 \mathrm{~h}$ 反应结束, 加入 $25 \mathrm{~mL}$ 二氯甲烷和 15 $\mathrm{mL}$ 水, 分出有机层, 水层用二氯甲烷萃取 $(20 \mathrm{~mL} \times 3)$, 合并有机相, 用无水硫酸钠干燥, 减压浓缩, 剩余物用 硅胶柱层析[洗脱剂: $V$ (石油醚) $: V($ 乙酸乙酯 $)=3: 1$ ] 分 离, 得 $42 \mathrm{mg}$ 绿色固体 10 (0.059 mmol, 28\%)和 $11 \mathrm{mg}$ 墨 绿色固体 11 (0.017 mmol, 8\%).

10: m.p. $241 \sim 244{ }^{\circ} \mathrm{C}$; UV-Vis $\left(\mathrm{CH}_{2} \mathrm{Cl}_{2}\right) \lambda_{\max }$

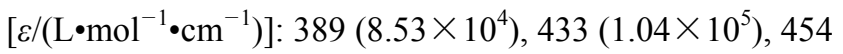
$\left(9.46 \times 10^{4}\right), \quad 590 \quad\left(2.81 \times 10^{4}\right), \quad 646 \quad\left(3.74 \times 10^{4}\right), \quad 706$ $\left(1.04 \times 10^{5}\right) \mathrm{nm} ;{ }^{1} \mathrm{H}$ NMR $\left(\mathrm{CDCl}_{3}\right) \delta:-0.28($ br s, $1 \mathrm{H}$, $\mathrm{NH}), 0.70$ (br s, $1 \mathrm{H}, \mathrm{NH}), 1.58$ (t, $J=7.6 \mathrm{~Hz}, 3 \mathrm{H}, 8 \mathrm{a}-\mathrm{CH}_{3}$ ), 1.77 (1.73) (d, $\left.J=7.2 \mathrm{~Hz}, 3 \mathrm{H}, 18-\mathrm{CH}_{3}\right), 2.18 \sim 2.42(\mathrm{~m}$, $2 \mathrm{H} .17 \mathrm{a}+17 \mathrm{bH}$ ), $2.55 \sim 2.70$ (m, 2H. $17 \mathrm{a}+17 \mathrm{~b}-\mathrm{H}), 3.45$ (q, $J=7.6 \mathrm{~Hz}, 2 \mathrm{H}, 8 \mathrm{a}-\mathrm{H}), 2.78$ (2.75), 3.10, 3.29, 3.69 (3.65) (each s, each 3H, $\mathrm{CH}_{3}+\mathrm{OCH}_{3}$ ), 4.19 (4.17) (d, $J=$ $9.0 \mathrm{~Hz}, 1 \mathrm{H}, 17-\mathrm{H}$ ), 4.33 (q, $J=7.4 \mathrm{~Hz}, 1 \mathrm{H}, 18-\mathrm{H}), 5.41$ (5.36) (s, 2H, 12-1'- $\left.\mathrm{CH}_{2}\right), 5.70$ (5.64) (d, $J=20.0 \mathrm{~Hz}, 1 \mathrm{H}$, $\left.13^{2}-\mathrm{H}\right), 5.80(5.55)\left(\mathrm{d}, J=20.0 \mathrm{~Hz}, 1 \mathrm{H}, 13^{2}-\mathrm{H}\right), 6.17(\mathrm{~d}$, $J=11.6,1 \mathrm{H}$, cis-3b-H), 6.24 (d, $J=18.0,1 \mathrm{H}$, trans $-3 \mathrm{~b}-\mathrm{H})$, 7.84 (dd, $J=18.0,11.6 \mathrm{~Hz}, 1 \mathrm{H}, 3 \mathrm{a}-\mathrm{H}), 8.32$ (8.31), 8.88 (8.87), 9.02 (each s, each $1 \mathrm{H}$, meso-H); ${ }^{13} \mathrm{C}$ NMR $(400 \mathrm{~Hz}$, $\left.\mathrm{CDCl}_{3}\right) \delta: 173.62,148.71,143.43,135.80,135.37,132.46$, $131.90,130.77,130.25,129.39,129.36,128.80,126.40$, 123.81, 121.88, 104.92, 101.43, 93.40, 51.85, 51.40, 48.75, 31.01, 30.09, 23.66, 23.60, 19.27, 17.41, 14.13, 11.90, 10.98; IR (KBr) v: $3409(\mathrm{~N}-\mathrm{H}), 2960(\mathrm{C}-\mathrm{H}), 1729,1700$ $(\mathrm{C}=\mathrm{O}), 1647(\mathrm{C}=\mathrm{C}), 1550$ (chlorin skeleton), 1462, 1376, 1211, 1143, $1053 \mathrm{~cm}^{-1}$; EI-MS $m / z: 703.3\left(\mathrm{M}+\mathrm{H}^{+}\right)$. Anal. calcd for $\mathrm{C}_{42} \mathrm{H}_{38} \mathrm{~N}_{8} \mathrm{O}_{3}$ : C 71.78, H 5.45, N 15.94; found C 71.89, H 5.39, N 16.06.

11: m.p. $237 \sim 239{ }^{\circ} \mathrm{C}$; UV-Vis $\left(\mathrm{CH}_{2} \mathrm{Cl}_{2}\right) \lambda_{\max }$

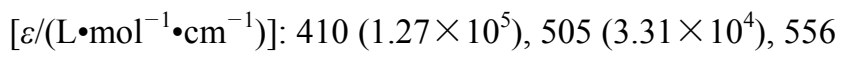
$\left(2.54 \times 10^{4}\right), 617\left(2.16 \times 10^{4}\right), 674\left(6.73 \times 10^{4}\right) \mathrm{nm} ;{ }^{1} \mathrm{H}$ NMR $\left(\mathrm{CDCl}_{3}\right) \delta:-0.73$ (br s, $\left.1 \mathrm{H}, \mathrm{NH}\right), 0.50($ br s, $1 \mathrm{H}$, $\mathrm{NH}), 1.63\left(\mathrm{t}, J=7.6 \mathrm{~Hz}, 3 \mathrm{H}, 8 \mathrm{a}-\mathrm{CH}_{3}\right), 1.78(\mathrm{~d}, J=7.2 \mathrm{~Hz}$, $\left.3 \mathrm{H}, 18-\mathrm{CH}_{3}\right), 2.23 \sim 2.38(\mathrm{~m}, 2 \mathrm{H}, 17 \mathrm{a}+17 \mathrm{~b}-\mathrm{H}), 2.51 \sim$ $2.71(\mathrm{~m}, 2 \mathrm{H}, 17 \mathrm{a}+17 \mathrm{~b}-\mathrm{H}), 3.52$ (q, $J=7.6 \mathrm{~Hz}, 2 \mathrm{H}, 8 \mathrm{a}-\mathrm{H})$, 2.76, 3.14, 3.33, 3.64 (each s, each $3 \mathrm{H}, \mathrm{CH}_{3}+\mathrm{OCH}_{3}$ ), 4.20 (d, $J=8.4 \mathrm{~Hz}, 1 \mathrm{H}, 17-\mathrm{H}), 4.39$ (q, $J=7.2 \mathrm{~Hz}, 1 \mathrm{H}, 18-\mathrm{H}$ ), $5.08\left(\mathrm{~d}, J=18.4 \mathrm{~Hz}, 13^{2}-\mathrm{H}\right), 5.24(\mathrm{~d}, J=18.4 \mathrm{~Hz}, 1 \mathrm{H}$, $\left.13^{2}-\mathrm{H}\right), 5.44$ (s, $\left.2 \mathrm{H}, 12-1^{\prime}-\mathrm{CH}_{2}\right), 6.17$ (d, $J=11.4 \mathrm{~Hz}, 1 \mathrm{H}$, cis-3b-H), 6.26 (d, $J=17.8 \mathrm{~Hz}, 1 \mathrm{H}$, trans-3b-H), 7.89 (dd, $J=17.8,11.4 \mathrm{~Hz}, 1 \mathrm{H}, 3 \mathrm{a}-\mathrm{H}$ ), 8.39, 9.12, 9.20 (each s, each $1 \mathrm{H}$, meso-H); ${ }^{13} \mathrm{C}$ NMR $\left(400 \mathrm{~Hz}, \mathrm{CDCl}_{3}\right) \delta: 203.01$, $197.56,173.51,171.36,161.10,155.49,150.74,148.98$, $145.07,141.51,137.84,136.41,136.09,135.86,131.76$, $131.09,130.04,129.15,128.31,128.09,126.41,122.57$, 109.10, 104.08, 97.20, 93.11, 56.67, 51.70, 50.96, 50.13, $39.12,31.02,30.00,25.82,23.43,19.45,17.44,12.11$, 11.21; IR (KBr) $v: 3454(\mathrm{~N}-\mathrm{H}), 2858(\mathrm{C}-\mathrm{H}), 1739,1700$ $(\mathrm{C}=\mathrm{O}), 1618(\mathrm{C}=\mathrm{C}), 1461$ (chlorin skeleton), 1448, 1380, 1253, 1172, 1083, 1010, $801 \mathrm{~cm}^{-1}$; EI-MS $\mathrm{m} / z$ : 656.2 $\left(\mathrm{M}+\mathrm{H}^{+}\right)$. Anal. calcd for $\mathrm{C}_{39} \mathrm{H}_{38} \mathrm{~N}_{6} \mathrm{O}_{4}: \mathrm{C} 71.54, \mathrm{H}$ 5.85, N 12.84; found C 71.41, H 5.69, N 12.89 .

$3.913^{1}$-二氭亚甲基- $13^{2}$-氧代- $13^{1}$-去氧焦脱镁叶绿酸a 甲酯(13)和 $13^{1}$-二氭亚甲基-12-甲氧甲酰基-12-去甲 基-13 ${ }^{1}$-去氧焦脱镁叶绿酸-a 甲酯(14)的合成

取 $220 \mathrm{mg}$ MPPa (1) (0.401 mmol)溶解于 $20 \mathrm{~mL}$ 无 水乙醇中, 加入过量的丙二睛 $(200 \mathrm{mg})$ 和 $0.5 \mathrm{~mL}$ 三乙 胺, 氮气保护下于 $80{ }^{\circ} \mathrm{C}$ 条件下搅拌反应 $4 \mathrm{~h}$, 减压除去 大部分溶剂后加入 $100 \mathrm{~mL}$ 水, 用二氯甲烷萃取 (100 $\mathrm{mL} \times 3)$, 合并有机层, 水洗后用无水硫酸钠干燥, 减压 浓缩, 剩余物用硅胶柱层析[洗脱剂: $V$ (石油醚) $: V($ 乙 酸乙酯 $)=3: 1$ ]分离出作为主要产物 12 的蓝绿色色带, 浓缩后重新溶解在 $20 \mathrm{~mL}$ 四氢呋喃中, 将反应体系温度 降低至 $0{ }^{\circ} \mathrm{C}$, 加入氢氧化锂溶液 $(400 \mathrm{mg} \mathrm{LiOH}$ 溶解在 $20 \mathrm{~mL}$ 甲醇和 $5 \mathrm{~mL}$ 水中), 室温搅拌 $6 \mathrm{~h}$, 用 $25 \%$ 的硫酸 溶液调至 $\mathrm{Ph}$ 值为 2 , 用 $\mathrm{CH}_{2} \mathrm{Cl}_{2}$ 萃取反应液 $(15 \mathrm{~mL} \times 3)$, 合并有机层, 水洗后用无水硫酸钠干燥, 减压浓缩, 剩 余物用硅胶柱层析 [洗脱剂: $V$ (石油醚) $: V$ (乙酸乙酯 $)=$ 3: 1] 分离, 得 $43 \mathrm{mg}$ 黄绿色固体 13 (0.072 mmol, 18\%) 和 $10 \mathrm{mg}$ 墨绿色固体 14 (0.016 mmol, 4\%).

13: m.p. $252 \sim 255{ }^{\circ} \mathrm{C}$; UV-Vis $\left(\mathrm{CH}_{2} \mathrm{Cl}_{2}\right) \lambda_{\max }$

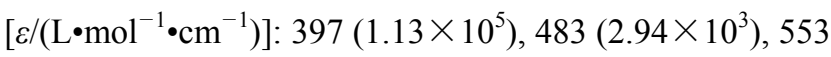
$\left(1.02 \times 10^{3}\right), 654\left(2.13 \times 10^{4}\right), 716\left(3.84 \times 10^{4}\right) \mathrm{nm} ;{ }^{1} \mathrm{H}$ NMR $\left(\mathrm{CDCl}_{3}\right) \delta:-1.98($ br s, $1 \mathrm{H}, \mathrm{NH}), 0.51($ br s, $1 \mathrm{H}$, $\mathrm{NH}), 1.70\left(\mathrm{t}, J=7.6 \mathrm{~Hz}, 3 \mathrm{H}, 8-\mathrm{CH}_{3}\right), 1.88(\mathrm{~d}, J=7.2 \mathrm{~Hz}$, $\left.3 \mathrm{H}, 18-\mathrm{CH}_{3}\right), 1.95 \sim 2.07(\mathrm{~m}, 1 \mathrm{H}, 17 \mathrm{a}+17 \mathrm{~b}-\mathrm{H}), 2.18 \sim$ $2.43(\mathrm{~m}, 2 \mathrm{H}, 17 \mathrm{a}+17 \mathrm{~b}-\mathrm{H}), 2.67 \sim 2.80(\mathrm{~m}, 1 \mathrm{H}, 17 \mathrm{a}+$ 17b-H), 3.35, 3.50, 3.51, 3.62 (each s, each 3H, $\mathrm{CH}_{3}+$ 
$\left.\mathrm{OCH}_{3}\right), 3.75(\mathrm{q}, J=7.6 \mathrm{~Hz}, 2 \mathrm{H}, 8 \mathrm{a}-\mathrm{H}), 5.11(\mathrm{~d}, J=8.0 \mathrm{~Hz}$, $1 \mathrm{H}, 17-\mathrm{H}), 4.63$ (q, $J=7.6 \mathrm{~Hz}, 1 \mathrm{H}, 18-\mathrm{H}), 6.30$ (dd, $J=$ 11.6, $1.0 \mathrm{~Hz}, 1 \mathrm{H}$, cis-3b-H), 6.36 (dd, $J=17.8,1.0 \mathrm{~Hz}, 1 \mathrm{H}$, trans-3b-H), 8.08 (dd, $J=17.8,11.6 \mathrm{~Hz}, 1 \mathrm{H}, 3 \mathrm{a}-\mathrm{H}), 8.94$, 9.50, 9.80 (each s, each 1H, meso-H); IR (KBr) v: 3429 $(\mathrm{N}-\mathrm{H}), 2914(\mathrm{C}-\mathrm{H})$, 1742, $1700(\mathrm{C}=\mathrm{O}), 1604(\mathrm{C}=\mathrm{C})$, 1529 (chlorin skeleton), 1422, 1388, 1304, 1126, 1010, 975 $\mathrm{cm}^{-1}$; EI-MS $m / z$ : $612.4\left(\mathrm{M}+\mathrm{H}^{+}\right)$. Anal. calcd for $\mathrm{C}_{37} \mathrm{H}_{34} \mathrm{~N}_{6} \mathrm{O}_{3}$ : C 72.77, H 5.61, N 13.76; found C 72.61, H $5.41, \mathrm{~N} 13.59$.

14: m.p. $227 \sim 229{ }^{\circ} \mathrm{C}$; UV-Vis $\left(\mathrm{CH}_{2} \mathrm{Cl}_{2}\right) \lambda_{\max }$

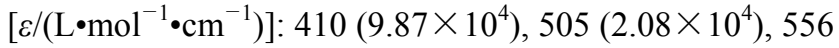
$\left(1.58 \times 10^{4}\right), 617\left(3.95 \times 10^{3}\right), 674\left(4.24 \times 10^{3}\right) \mathrm{nm} ;{ }^{1} \mathrm{H}$ NMR $\left(\mathrm{CDCl}_{3}\right) \delta: 0.05$ (br s, $\left.1 \mathrm{H}, \mathrm{NH}\right), 0.70$ br s, $1 \mathrm{H}, \mathrm{NH}$ ), $1.65\left(\mathrm{t}, J=7.6 \mathrm{~Hz}, 3 \mathrm{H}, 8-\mathrm{CH}_{3}\right), 1.74(\mathrm{~d}, J=7.2 \mathrm{~Hz}, 1 \mathrm{H}$, $\left.18-\mathrm{CH}_{3}\right), 1.98 \sim 2.06(\mathrm{~m}, 1 \mathrm{H}, 17 \mathrm{a}+17 \mathrm{~b}-\mathrm{H}), 2.15 \sim 2.36$ (m, $2 \mathrm{H}, 17 \mathrm{a}+17 \mathrm{~b}-\mathrm{H}), 2.50 \sim 2.67(\mathrm{~m}, 1 \mathrm{H}, 17 \mathrm{a}+17 \mathrm{~b}-\mathrm{H})$, 3.56 (q, $J=7.6 \mathrm{~Hz}, 2 \mathrm{H}, 8 \mathrm{a}-\mathrm{H}), 3.07,3.25,3.63,4.35$ (each s, each $\left.3 \mathrm{H}, \mathrm{OCH}_{3}+\mathrm{CH}_{3}\right), 4.08(\mathrm{~d}, J=9.0 \mathrm{~Hz}, 1 \mathrm{H}, 17-\mathrm{H})$, 4.27 (q, $J=7.2 \mathrm{~Hz}, 1 \mathrm{H}, 18-\mathrm{H}), 4.97(\mathrm{~d}, J=20.0 \mathrm{~Hz}, 1 \mathrm{H}$, $\left.13^{2}-\mathrm{H}\right), 5.13\left(\mathrm{~d}, J=20.0 \mathrm{~Hz}, 1 \mathrm{H}, 13^{2}-\mathrm{H}\right), 6.14$ (d, $J=11.6$ $\mathrm{Hz}, 1 \mathrm{H}$, cis-3b-H), $6.22(\mathrm{~d}, J=17.6 \mathrm{~Hz}, 1 \mathrm{H}$, trans-3b-H), 7.79 (dd, $J=17.6,11.6 \mathrm{~Hz}, 1 \mathrm{H}, 3 \mathrm{a}-\mathrm{H}$ ), 8.20, 8.87, 10.08 (each s, each $1 \mathrm{H}$, meso-H); IR $(\mathrm{KBr}) v: 3436(\mathrm{~N}-\mathrm{H})$, 2924, $2854(\mathrm{C}-\mathrm{H}), 1738,1698(\mathrm{C}=\mathrm{O}), 1622(\mathrm{C}=\mathrm{C})$, 1585 (chlorin skeleton), 1483, 1373, 1283, 1014, 912, 804 $\mathrm{cm}^{-1}$; EI-MS $\mathrm{m} / z$ : $640.7\left(\mathrm{M}+\mathrm{H}^{+}\right)$. Anal. calcd for $\mathrm{C}_{35} \mathrm{H}_{36} \mathrm{~N}_{6} \mathrm{O}_{4}$ : C 71.23, H 5.66, N 13.12; found $\mathrm{C} 71.37, \mathrm{H}$ $5.81, \mathrm{~N} 13.07$.

\subsection{0 体外光动力药理活性的测定}

\subsection{0 .1 待测样品和对照品}

分别选择化合物 $1,4,6 \sim 12$ 和 14 为待测化合物, 选 择临床光动力抗癌药物光敏素(Photofrin)和中位 meso四羟苯基二氢卟吩 (mTHPC) 为对照品. 将待测样品和对 照品用含 $20 \%$ 增溶剂聚氧乙烯菎麻油的水溶液溶解.

\subsection{0 .2 测定方法(MTT 比色检测法)}

在 $37{ }^{\circ} \mathrm{C} 、 5 \% \mathrm{CO}_{2}$ 、饱和湿度下, 小鼠肉瘤 S- 180 细胞贴壁培养于含 $10 \mathrm{~mL}$ 完全培养液 $(\alpha-\mathrm{MEM}$, 含 $10 \%$ 新生牛血清, $L$-谷氨酸盐, 青霉素以及链霉素)的细胞培 养瓶中. 细胞接种于含完全培养基的 96 孔细胞培养板 中, 每孔细胞密度为 $5 \times 10^{3}$ 个/孔. $37{ }^{\circ} \mathrm{C}$ 恒温培养过夜 后各孔分别加入不同浓度的光敏剂, 使其最终浓度分别 为 $10,1,0.1,0.05$ 以及 $0.01 \mu \mathrm{mol} \cdot \mathrm{L}^{-1}$, 在暗处 $37{ }^{\circ} \mathrm{C}$ 继续 恒温培养 $24 \mathrm{~h}$. 在光处理前用不含药物的完全培养基替 换孔中的培养基, 然后用 $670 \sim 700 \mathrm{~nm}$ 的光照射细胞 20 $\min$, 总光剂量为 $8.4 \mathrm{~J}$. 细胞在暗处于 $37{ }^{\circ} \mathrm{C}$ 继续恒温培 养 $48 \mathrm{~h}$. 在培养结束前 $4 \mathrm{~h}$, 分别在每孔中加入 $10 \mu \mathrm{L} 4.0$ $\mathrm{mg} / \mathrm{mL}$ 的 3-[2'-(4',5'-二甲基噻唑)]-2,5-二苯基四唑溴化 物(MTT)磷酸缓冲溶液. $4 \mathrm{~h}$ 后移除培养基和 MTT, 加入 $100 \mu \mathrm{L}$ DMSO 溶解甲暨结晶. 用酶标仪(Titertek Multiscan Plus MK II; Miles Inc.) 测定各孔在 $560 \mathrm{~nm}$ 的吸光 度. 每组实验重复三次, 标准误差均值 (SEMs) 小于 $10 \%$, 每次实验设 5 个重复孔. 以不加光敏剂和非光照 条件下的细胞为空白对照, 以光敏剂三次重复实验所得 吸光度平均值对光敏剂浓度作图得到各光敏剂的 $\mathrm{IC}_{50}$ 值. 结果分析及各化合物的 $\mathrm{IC}_{50}$ 值见表 1.

辅助材料(Supporting Information) 所有新化合物的 核磁共振氢谱数据. 这些材料可以免费从本刊网站 (http://sioc- journal.cn/)上下载.

\section{References}

[1] (a) Liu, C.; Dobhal, M. P.; Ethirajan, M.; Missert, J. R.; Pandey, R. K.; Balasubramanian, S.; Sukumaran, D. K.; Zhang, M.; Kadish, K. M.; Ohkubo, K.; Fukuzumi, S. J. Am. Chem. Soc, 2008, 130, 14311. (b) Machida, S.; Isoda, Y.; Kunieda, M.; Tamiaki, H. Tetrahedron Lett. 2012, 53, 6277.

(c) Fajer, J. Photosynth. Res. 2004, 80, 165.

(d) Bruckner, C.; McCarthy, J. R.; Daniell, H. W.; Pendon, Z. D.; Iiagan, R. P.; Francis, T. M.; Ren, L.; Birge, R. R.; Frank, H. A. Chem. Phys. 2003, 294, 285.

[2] (a) Chen, Y. H.; Li, G. L.; Pandey, R. K. Curr. Org. Chem. 2004, 8, 1105.

(b) Wang, J.-J. Chin. J. Org. Chem. 2005, 25, 1353 ( in Chinese). (王进军, 有机化学, 2005, 25, 1353.)

(c) Gil, M.; Bieniaszl, M.; Seshadri, M.; Fisher, D.; Ciesielski, M. J.; Chen, Y.; Pandey, R. K.; Kozbor, D. Brit. J. Cancer 2011, 103(10), 1.

(d) Ethirajan, M.; Joshi, P.; William, W. H.; Ohkubo, K.; Fukuzumi, S.; Pandey, R. K. Org. Lett, 2011, 8, 1956.

(e) Bellnier, D. A.; Greco, W. R.; Loewen, G. M.; Nava, H.; Oseroff, A. R.; Pandey, R. K.; Tsuchida, T.; Dougherty, T. J. Cancer Res. 2003, 63, 1806;

[3] (a) Ethirajan, M.; Joshi, P.; William, W. H.; Ohkubo, K.; Fukuzumi, S.; Pandey, R. K. Org. Lett. 2011, 13, 1956.

(b) Pavlov, V. Y.; Ponomarev, G. V. Chem. Heterocycl. Compd. 2004, 40, 393.

(c) Li, J.-Z.; Zhang, P.; Zhang, S.-G.; Yin, J.-G.; Wang, J.-J. Org. Biol. Chem. 2015, 13, 1992.

[4] (a) Pandey, S. K.; Zheng, X.; Morgan, J.; Missert, J. R.; Liu, T.-H.; Shibata, M.; Bellnier, D. A.; Oseroff, A. R.; Henderson, B. W.; Dougherty, T. J.; Pandey, R. K. Mol. Pharm. 2007, 4, 448.

(b) Kozyrey, A. N.; Chen, Y.-H.; Goswami, L. N.; Tabaczynaki, W. A.; Pandey, R. K. J. Org. Chem. 2006, 71, 1949.

(c) Li, J.-Z.; Zhang, P.; Yao, N.-N.; Zhao, L.-L.; Wang, J,-J.; Shim, Y.-K. Tetrahedron Lett. 2014, 55, 1086

[5] (a) Xu, X.-S.; Yao, N.-N.; Liu, Y.; Yin, J.-G.; Qi, C.-X.; Wang, J.-J. Chin. J. Org. Chem. 2014, 34, 938 (in Chinese).

(徐希森, 姚楠楠, 刘洋, 殷军港, 祁彩霞, 王进军, 有机化学, 2014, 34, 938.)

(b) Liu, Y.; Xu, X.-S.; Li, J.-Z.; Yin, J.-G.; Qi, C.-X.; Wang, J.-J. 
Chin. J. Org. Chem. 2014, 34, 552 ( in Chinese).

(刘洋, 徐希森, 李家柱, 殷军港, 祁彩霞, 王进军, 有机化学, 2014, 34, 552.)

(c) Sasaki, S.; Mizutani, K.; Kunieda, M.; Tamiaki, H. Tetrahedron 2011, 67, 6065 .

[6] (a) Wang, J.-J.; Wang, P.; Li, J.-Z.; Jakus, J.; Shin, Y.-K. Bull. Korean Chem. Soc. 2011, 32, 3473.

(b) Wang, J.-J.; Yin, Y.-F.; Yang, Z. J. Iran. Chem. Soc. 2013, 10, 583.

(c) Wang, J.-J.; Ji, J.-Y.; Han, G. F.; Wu, X.-R.; Wang, L.-M.; Shin, Y.-K. Acta Chim. Sinica 2004, 62, 302 (in Chinese).

(王进军, 纪建业, 韩光范, 邬旭然, 王鲁敏, 沈荣基, 化学学报, 2004, 62, 302.)

(d) Luca, A. R.; Giacomelli, G.; Porcheddu, A. J. Org. Chem. 2002, 67, 6272 .

[7] (a) Ji, J.-Y.; Wang, L. M.; Jing, J.-R.; Han, G.-F.; Wang, J. J. Chin. J. Org. Chem. 2007, 27, 493 (in Chinese).

(纪建业, 王鲁民, 荆济荣, 韩光范, 王进军, 有机化学, 2007, 27, 493.)

(b) Wang, J.-J.; Li, J.-Z.; Li, Y.-W.; Jakus, J.; Shim, Y.-K. J. Porphyrins Phthalocyanines 2010, 14, 859. (c) Wang, J.-J.; Li, J.-Z.; Jakus. J.; Shim, Y. K. J. Porphyrins Phthalocyanines 2012, 16, 122.

(d) Anilkumar, R.; Chandrsekhar, S. Tetrahedron Lett. 2001, 42, 5427.

[8] Zhang, Q. M.S. Thesis, Yantai University, Yantai, 2011 (in Chinese). (张千, 硕士论文, 烟台大学, 烟台, 2011.)

[9] Liu, Y. M.S. Thesis, Yantai University, Yantai, 2014 (in Chinese). (刘洋, 硕士论文, 烟台大学, 烟台, 2014.)

[10] (a) Arisawa, M.; Yanaguchi, M. Org. Lett. 2001, 3, 311.

(b) Kikugawa, Y.; Tsuji, C.; Miyazawa, E.; Sakamoto, T. Tetrahedron Lett. 2001, 42, 2337.

[11] (a) Ji, J.-Y.; Yin, J.-G.; Zhang, Q.; Liu, C.; Qi, C.-X.; Wang, J. J. Chin. J. Org. Chem. 2014, 34, 2047 (in Chinese).

(纪建业, 殷军港, 张千, 刘超, 祁彩霞, 王进军, 有机化学, 2014, 34, 2047.)

(b) Wang, L.-M.; Wang, P.; Liu, C.; Jin, Y.-X.; Wang, J.-J. Chin. J. Org. Chem. 2012, 32, 1700 (in Chinese).

(王鲁敏, 王朋, 刘超, 金英学, 王进军, 有机化学, 2012，32， 1700.)

[12] Smith, K. M.; Gogg, D. A.; Simpson, D. J. J. Am. Chem. Soc. 1985, 107, 4946.

(Li, L.; Fan, Y.) 